\title{
EVALUACIÓN DE UN RECUBRIMIENTO ANTICORROSIVO A BASE DE POLIESTIRENO EXPANDIDO RECICLADO
}

\author{
Evaluation of a recycled expanded polystyrene-based anticorrosive coat
}

\author{
${ }^{1}$ Mabel Parada Rivera, ${ }^{1}$ Tanya Cárdenas Valencia, , ${ }^{1,2}$ Paul Palmay Paredes, ${ }^{1}$ Danielita Borja Mayor- \\ ga, ${ }^{3}$ María Eugenia Ramos Flores
}

${ }^{1}$ Carrera de Ingeniería Química, Facultad de Ciencias, Escuela Superior Politécnica de Chimborazo

- ESPOCH. Riobamba, Ecuador.

${ }^{2}$ Universidad Rovira i Virgili, Tarragona, España.

${ }^{3}$ Departamento de Ciencias de la Tierra, Universidad Estatal Amazónica - UEA. Puyo, Ecuador.

*mparada@espoch.edu.ec

El desarrollo de recubrimientos anticorrosivos en la actualidad busca la reutilización de las grandes cantidades de polímeros desechados, entre los que destaca el poliestireno. Esta investigación tuvo como objetivo evaluar un recubrimiento anticorrosivo formulado en base a poliestireno expandido reciclado. Se desarrollaron dos formulaciones patrón con poliestireno expandido reciclado como resina, $\mathrm{D}$-limoneno como disolvente, $\mathrm{TiO} 2$ pigmento carga, $\mathrm{ZnO}$ agente anticorrosivo y octoato de cobalto como secante. Se evaluaron sus propiedades físicas, químicas y reológicas en referencia a la Norma Técnica Ecuatoriana NTE INEN 1045 "Pinturas anticorrosiva. Esmalte alquídico brillante. Requisitos”. Los resultados muestran que la formulación 2 da mejores resultados en función de su un tiempo de secado al tacto $(63,84 \mathrm{~min})$, viscosidad $(715,80 \mathrm{cP})$, porcentaje de adherencia $(96,00 \%)$, limpieza en vidrio y porcentaje de sólidos (39,50 \%). Adicionalmente, se comparó la resistencia a la corrosión del recubrimiento obtenido versus un recubrimiento comercial, mediante la exposición al ambiente salino de la playa las Palmas de la ciudad de Esmeraldas de placas de acero al carbono, acero inoxidable 304L y tol negro tratadas con ambos recubrimientos y aplicando la técnica de pérdida de peso. La velocidad de corrosión en las muestras donde se aplicó el recubrimiento de poliestireno, en milésimas de pulgadas por año (mpy), fueron 0,859 para el tol negro, 0,259 para acero al carbono y 0,064 para acero inoxidable 304L en base a la norma ADTM D-610 usando imágenes estandarizadas. Lo que evidencia el potencial del polímero reciclado como resina para recubrimiento anticorrosivo.

Palabras claves: Anticorrosivo, Corrosión, Poliestireno Expandido, Recubrimiento.

Development of anticorrosive coatings currently seeks the reuse of large quantities of discarded polymers, among which polystyrene stands out. The objective of this research was to evaluate an anticorrosive coating formulated based on recycled expanded polystyrene. Two standard formulations were developed with expanded polystyrene recycled as resin, D-limonene as solvent, $\mathrm{TiO} 2$ pigment charge, $\mathrm{ZnO}$ anticorrosive agent and cobalt octoate as a drying agent. Their physical, chemical and Theological properties were evaluated in reference to the Ecuadorian Technical Norm NTE INEN 1045 "Anticorrosive paints. Glossy alkyd enamel. Requirements. " The results show that the formulation 2 gives better results depending on its a time of drying to the touch $(63,840$ min), viscosity $(715,80 \mathrm{cP})$, percentage of adherence $(96,00 \%)$, cleaning in glass and percentage of solids (39,50\%). Additionally, the corrosion resistance of the obtained coating versus a commercial coating was compared by exposure, in the saline environment of Las Palmas Beach in the city of 
Esmeraldas, plates made of carbon steel, 304L stainless steel and black tol, all treated with both coatings and tested by weight loss technique. Corrosion rate in the samples where the polystyrene coating was applied, in thousandths of inches per year (mpy), were 0.859 for the black tol, 0.259 for carbon steel and 0.064 for 304L stainless steel. Also ASTM-610 standard standardized images was applied. Potential of the recycled polymer as a resin for anticorrosive coating was confirmed by the results of this investigation.

Key words: Anticorrosive, Corrosion, Expanded Polystyrene, Coating.

Fecha de recepción: 03-10-2018

Fecha de aceptación: 17-06-2019

\section{INTRODUCCIÓN}

La corrosión es uno de los principales problemas en las industrias química, petrolera, petroquímica, naval, automovilística, de construcción civil, edificaciones, vías, puentes, plataformas marinas, tuberías y represas, entre otras (1).

Esto lleva a importantes afectaciones en lo económico, con costos comprendidos entre el 2,00 y 4,00 \% del producto interno bruto de un país industrializado (2) (3).

El uso de recubrimientos inorgánicos es ampliamente utilizado para la prevención y control de la corrosión. Estos han demostrado un gran potencial sobre los recubrimientos orgánicos; sin embargo, han sido asociados con un impacto negativo sobre el medio ambiente y el ser humano, porque la mayoría de estos compuestos resultan ser muy tóxicos, costosos y dañinos (4). Esta situación ha creado la necesidad de encontrar anticorrosivos que sean ambientalmente amigables y de bajo costo (5).

La investigación busca determinar cómo responde a un ambiente salino un recubrimiento anticorrosivo a base de poliestireno expandido reciclado, para recabar información de la resistencia a la corrosión de piezas protegidas por aplicación superficial. Se busca así promover el planteamiento y desarrollo de una nueva formulación para alargar la vida útil de los materiales.

\section{MATERIALES Y MÉTODOS}

\section{Elaboración de recubrimiento de poliestireno expandi- do (EPS)}

Para la obtención del recubrimiento anticorrosivo se realizaron dos formulaciones en función de la cantidad de resina (poliestireno expandido), solvente y pigmentos agregados $\left(\mathrm{TiO}_{2}\right.$ y $\mathrm{ZnO}$, respectivamente), utilizando una cantidad constante de octoato de cobalto como aditivo. El proceso seguido fue el siguiente:

- Mezcla de 1 parte de resina con 0,50 partes de disolvente - Agitación a 300 rpm durante 30 minutos

- Añadir lentamente $\mathrm{TiO}_{2}$, hasta espesar

- Añadir las cargas de $\mathrm{ZnO}$, dependiendo de la absorción de aceite

- Adicionar solvente hasta ajustar la viscosidad

\section{Caracterización del recubrimiento}

Los parámetros para caracterizar el recubrimiento se seleccionaron en referencia a la norma NTE INEN 1045: "Pintura anticorrosiva esmalte alquídico brillante. Requisitos", que establecen los requisitos para pintura anticorrosiva (6).

Para la prueba de viscosidad se basó en la NTE INEN 1013 "Pinturas: Determinación de la viscosidad" (7), empleando un viscosímetro Brookfield. Todas las mediciones se efectuaron a $(25 \pm 1,0)^{\circ} \mathrm{C}$ y $45 \mathrm{rpm}$, usando la aguja $\mathrm{N}^{\circ}$ 3. La precisión de los resultados fue susceptible a la velocidad del viscosímetro y también al grado de viscosidad de la muestra.

El tiempo de secado determinó las etapas de formación de película en el curado del recubrimiento. La prueba se llevó a cabo siguiendo los lineamientos de la Norma ASTM D1640/D1640M (2018) "Standard Test Methods for Drying, Curing, or Film Formation of Organic Coatings", en una habitación bien ventilada, libre de corrientes de aire, polvo, productos de combustión y gases de labora- 
torio a una temperatura de $(23 \pm 2,0){ }^{\circ} \mathrm{C}$ y $(50 \pm 5,0) \%$ humedad relativa (8).

Para evaluar la adhesión de películas de recubrimiento a sustratos metálicos se desarrolló un método de prueba basado en la Norma ASTM D3359-17“Standard Test Methods for Rating Adhesion by Tape Test", donde al patrón se le hace seis u once cortes en cada dirección, se aplica sobre la red cinta sensible a la presión, que luego se retira. La adhesión se evalúa por comparación con descripciones e ilustraciones (9).

El porcentaje de sólidos, variable que indica la capacidad de relleno de una pintura o recubrimiento, se determinó en base a la Norma NTE INEN 1024 "Pinturas y productos afines. Determinación de materia no volátil y volátiles totales en pinturas"; consistiendo el ensayo en la evaporación de una alícuota apropiada, para posteriormente secar el residuo, pesarlo y calcular el porcentaje de material no volátil (10).

\section{Aplicación de los recubrimientos}

La aplicación de los recubrimientos elaborados siguió el método descrito por Arroyo y colaboradores (11): se realizaron sendas aplicaciones sobre placas de $50 \times 100 \times 3$ $\mathrm{mm}$ de acero al carbono, acero inoxidable 304L y tol negro. Las placas se limpiaron cuidadosamente y luego de la aplicación se dejaron secar por completo, removiendo parte de los recubrimientos en una de las caras para formar una " $\mathrm{X}$ " donde el material quedó expuesto al medio corrosivo.

El ensayo en el medio corrosivo se realizó siguiendo los lineamientos presentes en el libro de Protección de Materiales Metálicos, de Giudice (12): elaboración de probetas con los dos sistemas de recubrimientos, colocación en exposición natural en un proceso cíclico, con tiempo de exposición de 75 días (1800 h) en el entorno de la Playa de Las Palmas (ciudad de Esmeraldas, Ecuador), cuyas condiciones se indican en la Tabla 1

\begin{tabular}{cc}
\hline Características de la atmósfera & Datos promedios \\
\hline Temperatura & $27^{\circ} \mathrm{C}$ \\
Humedad relativa & $92 \%$ \\
Precipitaciones & $30 \%$ \\
Vientos & $5.4 \mathrm{~m} \mathrm{~s}^{-1}$ \\
pH & 8,20 \\
Concentración de sal & $24,00 \mathrm{~g} \mathrm{~L}^{-1}$
\end{tabular}

Tabla 1. Características de la estación Las Palmas (Esmeraldas). Fuente: INOCAR (2017).

\section{Caracterización de la corrosión}

Los dos parámetros utilizados para caracterizar la corrosión experimentada por las placas, y el efecto de los recubrimientos, fueron la "pérdida promedio de peso" y la "velocidad de corrosión".

La pérdida promedio de peso se calcula en base a la pérdida de masa de la placa metálica en el periodo de tiempo considerado, aplicando la siguiente ecuación. $P_{\text {perdido }}=P_{\text {inicial }}-P_{\text {final }}$

Dónde:

$\mathrm{P}_{\text {perdido }}$ : Peso perdido por corrosión, en gramos

$\mathrm{P}_{\text {inicial }}$ : Peso de la muestra, antes de la prueba, en gramos

$\mathrm{P}_{\text {final }}$ : Peso de la muestra, al final de la prueba, en gramos

La velocidad de corrosión (mpy) se determina de acuerdo a la ecuación

$m p y=\frac{W_{L} * 22273}{D^{*} A^{*} T}$

Dónde:

mpy: rango o velocidad de corrosión

$\mathrm{W}_{\mathrm{L}}$ : pérdida de peso sufrida por el testigo corrosimétrico

D: densidad del material en sobre centímetros cúbicos $(\mathrm{g} / \mathrm{cm} 3)$

A: área de exposición, en pulgadas cuadradas (in2)

T: tiempo de exposición del material al ambiente corrosivo en días

\section{Comparación recubrimiento de polies-} tireno expandido y recubrimiento comercial

Se comparó la eficiencia, bajo las mismas condiciones, del recubrimiento obtenido con respecto a un recubrimiento comercial, usando como referencia la norma ASTM D-610 "Standard Test Method for Evaluating Degree of Rusting on Painted Steel Surfaces" que cual permite evaluar el grado de oxidación en las superficies de acero recubiertas con una capa polimérica. En la tabla a continuación (Tabla 
2) se detalla la caracterización del grado de oxidación con base en el porcentaje de óxido en la superficie del acero, en una escala de 0 a 10; donde la distribución de la corrosión se puede diferenciar como "puntual", cuando la oxidación se concentra en pocas áreas localizadas, "general", cuando varias manchas de óxido se distribuyen en la superficie, y "dispersa”, cuando el óxido se distribuye como pequeñas partículas repartidas por toda la superficie.(13)

\begin{tabular}{|c|c|c|c|c|}
\hline \multirow{2}{*}{ Grado de corrosión } & \multirow{2}{*}{$\%$ de la superficie oxidada } & \multicolumn{3}{|c|}{ Ejemplos Visuales } \\
\hline & & Puntual (s) & General $(G)$ & Disperso $(P)$ \\
\hline 10 & Menor o igual a $0,01 \%$ & & & \\
\hline 9 & Entre el $0,01 \%$ a $0,03 \%$ & $9-s$ & $9-\mathrm{G}$ & 9-P \\
\hline 8 & Entre el $0,03 \%$ a $0,1 \%$ & $8-s$ & $8-\mathrm{G}$ & $8-\mathrm{P}$ \\
\hline 7 & Entre el $0,1 \%$ a $0,3 \%$ & $7-s$ & $7-\mathrm{G}$ & 7-P \\
\hline 6 & Entre el $0,3 \%$ a $1 \%$ & $6-s$ & $6-\mathrm{G}$ & $6-\mathrm{P}$ \\
\hline 5 & Entre el $1 \%$ a $3 \%$ & $5-s$ & $5-\mathrm{G}$ & $5-\mathrm{P}$ \\
\hline 4 & Entre el $3 \%$ a $10 \%$ & $4-s$ & $4-\mathrm{G}$ & $4-\mathrm{P}$ \\
\hline 3 & Entre el $10 \%$ a $16 \%$ & $3-s$ & $3-\mathrm{G}$ & $3-\mathrm{P}$ \\
\hline 2 & Entre el $16 \%$ a $33 \%$ & $2-s$ & $2-\mathrm{G}$ & $2-\mathrm{P}$ \\
\hline 1 & Entre el $33 \%$ a $50 \%$ & $1-s$ & $1-\mathrm{G}$ & $1-\mathrm{P}$ \\
\hline 0 & Mayor al $50 \%$ & & & \\
\hline
\end{tabular}

Tabla 2. Clasificación visual y descripción del grado de oxidación de una superficie. Fuente: ASTM D-610, 2008.

\section{RESULTADOS}

La formulación con mejores resultados se describen la tabla siguiente (Tabla 3):

\begin{tabular}{lccc}
\hline Componente & Sustancia & Cantidad & Unidad \\
\hline Resina & $\begin{array}{c}\text { Poliestireno } \\
\text { expandido } \\
\text { reciclado }\end{array}$ & 80,00 & $\mathrm{~g}$ \\
Anticorrosivo & $\mathrm{ZnO}$ & 13,50 & $\mathrm{~g}$ \\
Solvente & Limoneno & 200,00 & $\mathrm{~mL}$ \\
Pigmento & $\mathrm{TiO}_{2}$ & 8,70 & $\mathrm{~g}$ \\
Cargas & $\mathrm{CaCO}_{3}$ & 5,00 & $\mathrm{~g}$ \\
\hline
\end{tabular}

Tabla 3. Formulación para recubrimiento a base de poliestireno expandido reciclado.

Fuente: Autores

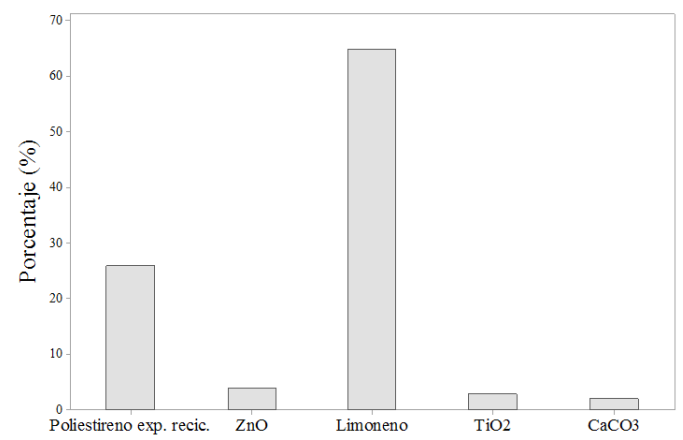

Figura 1. Composición porcentual del recubrimiento
Una vez determinada la formulación óptima para el recubrimiento, se verificó que esta cumpla los parámetros de viscosidad, adherencia, tiempo de secado y porcentaje de sólidos, según lo requerido en la norma INEN NTE 1045.

Los resultados se muestran a continuación (Tabla 4), además de indicar los valores máximo y mínimo, calculados mediante análisis estadístico.

\begin{tabular}{cccccc}
\hline Variable & $\begin{array}{c}\text { Normas } \\
\text { referencia }\end{array}$ & Valor norma & $\begin{array}{c}\text { Valor } \\
\text { obtenido }\end{array}$ & Mínimo & Máximo \\
\hline Viscosidad & $\begin{array}{c}\text { NTE INEN } \\
1010\end{array}$ & $\begin{array}{c}\text { Mínimo } 630 \\
\text { cP }\end{array}$ & $715,8 \mathrm{cP}$ & 715,0 & 716,6 \\
Adherencia & $\begin{array}{c}\text { INEN NTE } \\
1006\end{array}$ & $98 \%$ & $96 \%$ & 95,8 & 96,91 \\
$\begin{array}{c}\text { Tiempo de } \\
\text { secado }\end{array}$ & $\begin{array}{c}\text { INEN NTE } \\
1011\end{array}$ & Máximo $4 \mathrm{~h}$ & $\begin{array}{c}63,84 \\
\text { min }\end{array}$ & $\begin{array}{c}60,69 \\
\text { min }\end{array}$ & $\begin{array}{c}67,00 \\
\text { min }\end{array}$ \\
$\begin{array}{c}\text { Porcentaje } \\
\text { de sólidos }\end{array}$ & $\begin{array}{c}\text { NTE INEN } \\
1024\end{array}$ & $40 \%$ & $39,5 \%$ & 37,24 & 41,85 \\
\hline
\end{tabular}

Tabla 4. Datos de variables del recubrimiento.

Fuente: Autores

Luego de evaluar la resistencia a la corrosión se evidenció que la velocidad de corrosión (mpy) en las diferentes probetas varía en función del tiempo que están expuesta al medio corrosivo. 


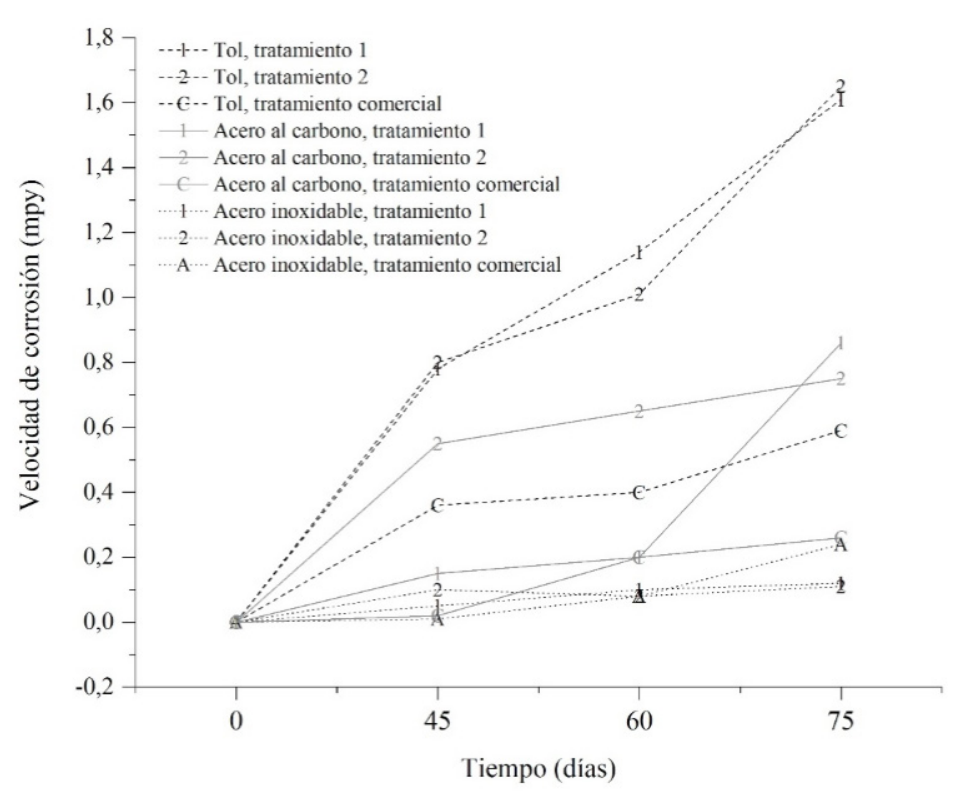

Figura 2: Comparativa de velocidades de corrosión, entre primera formulación "1", segunda formulación "2" y recubrimiento comercial "C".

Elaborado por: Autores

La placa que más cantidad de material pierde es la de tol negro cubierta con las formulaciones 1 y 2 , mientras que la placa de tol negro cubierta con el recubrimiento comercial presenta menor perdida de material en comparación a las otras formulaciones.

Con base en los resultados de la inspección visual, basada en la norma ASTM D610, se confirma que la formación de óxido superficial se incrementa a medida que el tiempo de exposición al ambiente salino aumenta.

\section{DISCUSIÓN}

Se encontró que la formulación óptima se logró al utilizar 200,0 mL de D-Limoneno como solvente, 13,5 g de ZnO como protector ante la formación de moho en la película, $8,7 \mathrm{~g}$ de $\mathrm{TiO}_{2}$ (pigmento), carbonato de calcio $5 \mathrm{~g}$ y octoato de cobalto como gente secante. Esto se explica no solo por lo analizado sino también por distintos investigadores como Chaieb et al.(5), quienes encontraron que el limoneno se adsorbe espontáneamente sobre la superficie del metal, y también Abdel Gabel et al. (14), halló que la presencia de $\mathrm{ZnO}$ o $\mathrm{TiO}_{2}$ tiene un efecto positivo en la eficiencia de la protección de las pinturas, destacando que el $\mathrm{ZnO}$ ofrece mayor resistencia a la corrosión que el $\mathrm{TiO}_{2}$.

Se realizaron ensayos de laboratorio regidos por procedimientos de nomas específicas como: Viscosidad (NTE INEN 1013), tiempo de secado (ASTM D640), adhesión (ASTM 3359) y porcentaje de solidos (NTE INEN 1024) con el fin de comparar los valores de las variables de calidad obtenidas con la Norma NTE INEN 1045:1984

Adicionalmente, se identificó la composición con la que se obtuvo los mejores resultados en la aplicación del recubrimiento. Los resultados de las caracterización físico-química y reológica del recubrimiento revelaron los siguientes valores promedio: 715,8 cP de viscosidad; $98 \%$ de adhesión, 2 hora de tiempo de secado y $39,5 \%$ de sólidos, siendo particularmente útil para la investigación los datos de porcentaje de adhesión y la cantidad de sólidos; puesto que permitieron identificar tanto el grado de unión entre la película y el material base como la cantidad de materia no volátil en el recubrimiento, es decir el residuo de pintura que queda después del proceso de secado total de la película.

Posteriormente para la evaluación de la velocidad de corrosión, expresada en mili pulgadas por año (mpy), se observó que las probetas cubiertas por la primera formulación experimentaban mayor pérdida de peso en comparación con la segunda formulación; sin embargo, éstas al ser comparadas con las pintadas por el recubrimiento comercial presentaban casi similares perdidas de peso; lo que podría atribuirse a los reactivos usados para la obtención del recubrimiento.

La comparación de las superficies tratadas con las formulaciones se realizó siguiendo la norma ASTM D610 "Standard Test Method for Evaluating Degree of Rusting on Painted Steel Surfaces" mediante inspección visual de la superficie por debajo de los recubrimientos. Se determinó que la segunda formulación presentó el mejor comportamiento de control de la corrosión, con formación de óxido puntual en pocas áreas localizadas; en tanto que en la lámina tratada con recubrimiento comercial surgió 
oxidación dispersa visible como formulación comercial.

pequeñas partículas distribuidas por toda su superficie.

\section{CONCLUSIONES}

Los tiempos de secado, viscosidades, porcentajes de adherencia y de sólidos de las formulaciones obtenidas cumplieron lo normado por la INEN NTE 1045. La formulación óptima se obtiene utilizando $80 \mathrm{~g}$ de poliestireno expandido reciclado, $200 \mathrm{~mL}$ de D-Limoneno, 13,5 g de óxido de zinc, $8,7 \mathrm{~g}$ de óxido de titanio, $5 \mathrm{~g}$ de carbonato de calcio y $2,00 \%$ de octoato de cobalto. Dicha formulación es la que logró mayor resistencia a la corrosión, con rendimiento superior al de la

Las probetas de tol negro, acero al carbono y acero inoxidable $304 \mathrm{~L}$ mostraron velocidades de corrosión promedio, en milésimas de pulgada por año, de 0,859 ; 0,259; 0,064 respectivamente, siendo en el caso del acero inoxidable donde las mpy de los diferentes recubrimientos presentaron menor variación; en el caso de acero al carbono hubo mayor diferencia entre la efectividad de las formulaciones estudiadas con respecto al recubrimiento comercial.

La inspección visual, de acuerdo a la norma ASTM D-610, identificó a la segunda formulación elaborada como la que desarrolla mejor función anticorrosiva.

Los resultados confirman el potencial del polímero reciclado como resina aplicable para tratamientos anticorrosivos.

\section{$\mathrm{R}$}

1. Meza Castellar P, Tejada Tovar C, Benítez Contreras I, Vélez Díaz J, Villabona Ortiz A. Uso de poliestireno expandido reciclado para la obtención de un recubrimiento anticorrosivo [Internet]. Vol. 11, Producción + Limpia. Corporacion Universitaria Lasallista, Facultad de Ingenierias; 2016 [cited 2019 Jan 2]. 13-21 p. Available from: http://www.scielo.org.co/scielo.php?pi$\mathrm{d}=$ S1909-04552016000100002\&script=sci_abstract\&tlng=es

2. Rosario Francia S, Yácono Llanos JC. Materiales: la corrosión, su tradición y alcances. Rev del Inst Investig la Fac Ing Geológica, Minera, Metal y Geográfica [Internet]. 2012 Jan 28;6(11):71-7. Available from: http://revistasinvestigacion.unmsm.edu.pe/index.php/iigeo/article/view/751

3. Revie RW, Uhlig HH. Uhlig's corrosion handbook. 3rd ed. Revie RW, editor. Ottawa: Wiley; 2011. 1253 p.

4. Contreras IB, Díaz JV, Ortiz AV. Uso de poliestireno expandido reciclado para la obtención de un recubrimiento anticorrosivo *. 2016;11(1):13-21.

5. Chaieb E, Bouyanzer A, Hammouti B, Benkaddour M. Inhibition of the corrosion of steel in 1 M HCl by eugenol derivatives. Appl Surf Sci [Internet]. 2005 Jun 15 [cited 2019 Jan 2];246(1-3):199206. Available from: https://www.sciencedirect.com/science/article/pii/S0169433204015533

6. Instituto Ecuatoriano de Normalización. NTE INEN 1045: Pinturas anticorrosiva. Esmalte alquídico brillante. Requisitos [Internet]. INEN, editor. Quito: INEN; 1983 [cited 2019 Jan 6]. 8 p. Available from: https://archive.org/stream/ec.nte.1045.1984\#page/n1/mode/2up

7. Instituto Ecuatoriano de Normalización. NTE INEN 1013: Pinturas. Determinación de la viscosidad [Internet]. Quito: INEN; 1983 [cited 2019 Jan 6]. 12 p. Available from: https://archive.org/ stream/ec.nte.1013.1984?ui=embed\#page/n1/mode/2up

8. ASTM International. ASTM D1640 / D1640M - 14(2018) Standard Test Methods for Drying, Curing, or Film Formation of Organic Coatings [Internet]. West Conshohocken, PA.: ASTM; 2018 [cited 2019 Jan 7]. 8 p. Available from: https://www.astm.org

9. ASTM International. ASTM D3359 - 17 Standard Test Methods for Rating Adhesion by Tape Test [Internet]. West Conshohocken, PA: ASTM; 2017 [cited 2019 Jan 7]. Available from: https:// www.astm.org/

10. Instituto Ecuatoriano de Normalización. NTE INEN 1024: Pinturas y productos afines. Deter- 
minación de materia no volátil y volátiles totales en pinturas [Internet]. Quito: INEN; 1998 [cited 2019 Jan 7]. Available from: https://archive.org/details/ec.nte.1024.1998/page/n5

11. Arroyo Páramo G, Aguilar Márquez A, Ortiz Valera JA, Sánchez Pérez F, Ortiz Prado A. Evaluación de dos pinturas en el acero SAE 1010, utilizando formulaciones de ácido tánico y fosfórico como convertidores de óxidos. In: XVII Congreso Internacional Anual de la SOMIM [Internet]. San Luis Potosí: SOMIM; 2011 [cited 2019 Jan 15].p. A3_39. Available from: http://somim.org.mx/ memorias/memorias2011/pdfs/A3/A3_39.pdf

12. Giudice CA, Canosa G. Protección de Materiales Metálicos [Internet]. 1st ed. Buenos Aires: Universidad Tecnológica Nacional; 2017 [cited 2019 Jan 15]. 274 p. Available from: http://www. edutecne.utn.edu.ar

13. ASTM International. ASTM D610-08(2008), Standard Practice for Evaluating Degree of Rusting on Painted Steel Surfaces [Internet]. West Conshohocken, PA; 2008. Available from: www. astm.org

14. Abdel-Gaber AM, Nabey BAA-E, Khamis E, Abdelattef OA, Aglan H, Ludwick A. Influence of natural inhibitor, pigment and extender on corrosion of polymer coated steel. Prog Org Coatings [Internet]. 2010 Dec 1 [cited 2019 Feb 26];69(4):402-9. Available from: https://www.sciencedirect. com/science/article/pii/S0300944010002274 